PROCEEDINGS OF THE

AMERICAN MATHEMATICAL SOCIETY

Volume 135, Number 8, August 2007, Pages 2549-2554

S 0002-9939(07)08762-X

Article electronically published on February 6, 2007

\title{
A MULTILINEAR PHELPS' LEMMA
}

\author{
RICHARD ARON, ANTONIA CARDWELL, DOMINGO GARCÍA, \\ AND IGNACIO ZALDUENDO
}

(Communicated by N. Tomczak-Jaegermann)

\begin{abstract}
We prove a multilinear version of Phelps' Lemma: if the zero sets
\end{abstract} of multilinear forms of norm one are 'close', then so are the multilinear forms.

\section{INTRODUCTION}

Let $X$ denote a Banach space, and $S_{X}$ its unit sphere. The following two results are both simple and well-known.

Theorem A. If $f_{j}, g: X \rightarrow \mathbb{C}, j=1, \ldots, k$, are linear forms, then

$$
\bigcap_{j=1}^{k} \operatorname{Ker} f_{j} \subset \operatorname{Ker} g \Rightarrow g=\sum_{j=1}^{k} a_{j} f_{j} \text { for some } a_{1}, \ldots, a_{k} \in \mathbb{C} \text {. }
$$

Theorem B. If $f, g: X \rightarrow \mathbb{C}$ are linear forms of norm one, then

$$
\text { Ker } f \subset K e r g \Rightarrow g=\text { af for some }|a|=1 \text {. }
$$

In 1960, R. Phelps [8] proved a continuous version of Theorem B. This result has come to be known as Phelps' Lemma, and also as the Parallel Hyperplane Lemma. It has had several important applications, including a fundamental role in the proof of the Bishop-Phelps Theorem 3. It has also been used to prove that, for any real Banach space $X$, the kernel of every $x^{* *} \in X^{* *} \backslash X$ is a norming hyperplane in $X^{*}\left[\right.$ [] , and to provide an estimate for the distance from $x^{* *}$ to $X[7$. Recall that the natural generalization of the Bishop-Phelps Theorem to multilinear mappings is false, in general [1. Nevertheless, Phelps' Lemma can be extended to the multilinear setting. The purpose of the present paper is to provide a proof of this.

Since our argument will refer to the proof of Phelps' Lemma, we include it here. (Note that a recent, more geometric, proof of this result was given by the second author in [5].)

Phelps' Lemma. If $f, g: X \rightarrow \mathbb{C}$ are linear forms of norm one and $\varepsilon>0$, then

$$
S_{X} \cap\{f(x)=0\} \subset S_{X} \cap\{|g(x)| \leq \varepsilon\} \Rightarrow\|g-\alpha f\| \leq 2 \varepsilon \text { for some }|\alpha|=1 .
$$

Received by the editors February 9, 2006 and, in revised form, April 11, 2006.

2000 Mathematics Subject Classification. Primary 46B20; Secondary 47A07.

Key words and phrases. Phelps' Lemma, multilinear forms.

The first and third authors were partially supported by MEC and FEDER Project MTM200508210 .

The fourth author was supported by a Fulbright Commission grant.

(C)2007 American Mathematical Society Reverts to public domain 28 years from publication 
Proof. Consider $\left.g\right|_{K e r f}$. Since the norm of $\left.g\right|_{K e r f}$ on that subspace is less than $\varepsilon$, we can use the Hahn-Banach Theorem to conclude that there is an $h \in X^{\prime}$ such that $\|h\| \leq \varepsilon$ and $h=g$ on $\operatorname{Ker} f$. We have that $\operatorname{Ker} f \subset \operatorname{Ker}(g-h)$, so by Theorem A, $g-h=a f$ for some $a \in \mathbb{C}$. Thus $g-a f=h$, and $\|g-a f\|=\|h\| \leq \varepsilon$.

Now $|1-| a||=|\|g\|-\|a f\|| \leq\|g-a f\| \leq \varepsilon$, so $1-\varepsilon \leq|a| \leq 1+\varepsilon$. If we now take $\alpha=\frac{a}{|a|}$, then $\|g-\alpha f\|=\|g-a f+(a-\alpha) f\| \leq\|g-a f\|+|a-\alpha| \leq 2 \varepsilon$.

Clearly, as $\varepsilon \rightarrow 0$, one recovers Theorem B. Note also that if we are not concerned about the size of the constant $a$, then the first paragraph of the above proof shows that $\|g-a f\| \leq \varepsilon$. We shall refer to this as the first part of Phelps' Lemma. Note also that the second part of Phelps' Lemma, which provides the $2 \varepsilon$ estimate, follows immediately from $\|g-a f\| \leq \varepsilon$ regardless of the fact that $f$ and $g$ are linear forms. We shall use this below for multilinear forms.

It is interesting to note that Theorem A above does not hold for multilinear forms, although Theorem B does [2].

Our proof of a multilinear Phelps' Lemma will require the use of constants $c_{k}>0$ such that if $P$ and $Q$ are $k$-homogeneous polynomials, then

$$
c_{k}\|P\|\|Q\| \leq\|P Q\| \text {. }
$$

The existence of such constants has been observed by C. Benítez, Y. Sarantopoulos, and A. Tonge 4 and also by R. Ryan and B. Turett [9].

Although we write all our proofs for complex Banach spaces, note that the same arguments remain true in the real case, by simply replacing $\mathbb{C}$ by $\mathbb{R}$.

We would like to thank Vicente Montesinos for several useful comments regarding applications of Phelps' Lemma.

\section{A multilinear version of Phelps' Lemma}

We will denote by $X_{1}, \ldots, X_{n}$ complex Banach spaces with $S_{X_{i}}$ being the associated unit spheres. We will use the notation $x=\left(x_{1}, \ldots, x_{n-1}\right) \in X_{1} \times \cdots \times X_{n-1}$ and $y \in X_{n}$. $A$ and $B$ will denote $n$-linear forms on $X_{1} \times \cdots \times X_{n}$ of norm one, and we will write $A(x, y)=A\left(x_{1}, \ldots, x_{n-1}, y\right) . A_{x}: X_{n} \rightarrow \mathbb{C}$ will be the linear function obtained by fixing $x$, and $A_{y}: X_{1} \times \cdots \times X_{n-1} \rightarrow \mathbb{C}$ will be the $n-1$-linear function obtained by fixing $y$ (and similarly for $B$ ). For subsets $D \subset S_{X_{1}} \times \cdots \times S_{X_{n}}$, denote $D_{x}=\left\{y \in S_{X_{n}}:(x, y) \in D\right\}, D_{y}=\left\{x \in S_{X_{1}} \times \cdots \times S_{X_{n-1}}:(x, y) \in D\right\}$. We will also write

$$
Z(A)=\left\{(x, y) \in S_{X_{1}} \times \cdots \times S_{X_{n}}: A(x, y)=0\right\},
$$

and for any $\varepsilon>0$,

$$
\varepsilon(B)=\left\{(x, y) \in S_{X_{1}} \times \cdots \times S_{X_{n}}:|B(x, y)| \leq \varepsilon\right\} .
$$

Also,

and

$$
Z\left(A_{y}\right)=\left\{x \in S_{X_{1}} \times \cdots \times S_{X_{n-1}}: A_{y}(x)=0\right\}
$$

$$
\varepsilon\left(B_{y}\right)=\left\{x \in S_{X_{1}} \times \cdots \times S_{X_{n-1}}:\left|B_{y}(x)\right| \leq \varepsilon\right\}
$$

We begin with an algebraic result due to [2]. The proof presented here is different, shorter, and has a bearing on our later generalization of Phelps' Lemma.

Proposition 1.1. If $A$ and $B$ are $n$-linear forms of norm one, then

$$
Z(A) \subset Z(B) \Rightarrow B=\alpha A \text { for some }|\alpha|=1 \text {. }
$$


Proof. We proceed by induction on $n$. For $n=1$, this is Theorem B. For $n>1$, suppose the result true for $n$-1-linear forms. We have, for each $y \in S_{X_{n}}$,

$$
Z\left(A_{y}\right)=Z(A)_{y} \subset Z(B)_{y}=Z\left(B_{y}\right) .
$$

Thus by the induction hypothesis, $B_{y}=\alpha_{y} A_{y}$ for some $\alpha_{y} \in \mathbb{C}$. Also, for each $x \in S_{X_{1}} \times \cdots \times S_{X_{n-1}}$,

$$
Z\left(A_{x}\right)=Z(A)_{x} \subset Z(B)_{x}=Z\left(B_{x}\right),
$$

so by Theorem B, $B_{x}=\alpha_{x} A_{x}$ for some $\alpha_{x} \in \mathbb{C}$. Note that given any $(x, y)$ with $A(x, y) \neq 0$,

$$
\alpha_{y}=\frac{B(x, y)}{A(x, y)}=\alpha_{x} .
$$

Consider $y_{1}$ and $y_{2}$ such that $A_{y_{1}} \neq 0 \neq A_{y_{2}}$. Then $\alpha_{y_{1}}=\alpha_{y_{2}}$. Indeed, taking $x \notin Z\left(A_{y_{1}}\right) \cup Z\left(A_{y_{2}}\right)$, we have $\alpha_{y_{1}}=\alpha_{x}=\alpha_{y_{2}}$. So for $y$ 's such that $A_{y} \neq 0$, setting $\alpha=\alpha_{y}$ produces a well-defined constant. If $y$ is such that $A_{y}=0$, then by hypothesis $B_{y}=0$, so $B_{y}=\alpha A_{y}$ in this case as well. Thus $B_{y}=\alpha A_{y}$ for all $y$, so $B=\alpha A$. Since both have norm one, $|\alpha|=1$.

Our multilinear Phelps' Lemma will be a continuous version of Proposition 1.1: if $Z(A) \subset \varepsilon(B)$, then $B \approx \alpha A$ for some $\alpha$ with $|\alpha|=1$. For each $\varepsilon>0, n>1$, $x \in S_{X_{1}} \times \cdots \times S_{X_{n-1}}$, and $y \in S_{X_{n}}$ we shall need the following sets, which generalize the singletons $\left\{\alpha_{y}\right\}$ in the previous proof:

$$
\begin{aligned}
& \Lambda_{x}(\varepsilon)=\left\{\beta \in \mathbb{C}:\left\|B_{x}-\beta A_{x}\right\| \leq \varepsilon\right\}, \\
& \Lambda_{y}(\varepsilon)=\left\{\alpha \in \mathbb{C}:\left\|B_{y}-\alpha A_{y}\right\| \leq \varepsilon\right\} .
\end{aligned}
$$

Lemma 1.2. If $Z(A) \subset \varepsilon(B)$, then $\Lambda_{x}(\varepsilon)$ is non-empty for all $x \in S_{X_{1}} \times \cdots \times$ $S_{X_{n-1}}$.

Proof. For all $x$,

$$
Z\left(A_{x}\right)=Z(A)_{x} \subset \varepsilon(B)_{x}=\varepsilon\left(B_{x}\right) .
$$

If $B_{x}=0$, any $\beta$ with $|\beta| \leq \varepsilon$ belongs to $\Lambda_{x}(\varepsilon)$. If $A_{x}=0$, then $\left\|B_{x}\right\| \leq \varepsilon$, and $\left\|B_{x}-\beta A_{x}\right\| \leq \varepsilon$ for all $\beta \in \mathbb{C}$, so $\Lambda_{x}(\varepsilon)=\mathbb{C}$. Suppose then that both are non-zero, and normalize:

$$
Z\left(\frac{A_{x}}{\left\|A_{x}\right\|}\right)=Z\left(A_{x}\right) \subset \varepsilon\left(B_{x}\right)=\frac{\varepsilon}{\left\|B_{x}\right\|}\left(\frac{B_{x}}{\left\|B_{x}\right\|}\right) .
$$

By the first part of Phelps' Lemma, there is a $\beta$ for which

$$
\left\|\frac{B_{x}}{\left\|B_{x}\right\|}-\beta \frac{A_{x}}{\left\|A_{x}\right\|}\right\| \leq \frac{\varepsilon}{\left\|B_{x}\right\|} \text {. }
$$

Thus $\left\|B_{x}-\beta \frac{\left\|B_{x}\right\|}{\left\|A_{x}\right\|} A_{x}\right\| \leq \varepsilon$. So $\beta \frac{\left\|B_{x}\right\|}{\left\|A_{x}\right\|} \in \Lambda_{x}(\varepsilon)$.

Note that we have not yet proved that the sets $\Lambda_{y}(\varepsilon)$ are non-empty. Of course for bilinear $A$ this is true by the above lemma, but for $n>2$, this is an essential part of Theorem 1.4 below.

Lemma 1.3. If $Z(A) \subset \varepsilon(B)$, then

i) If $\varepsilon \leq \varepsilon^{\prime}$, then $\Lambda_{y}(\varepsilon) \subset \Lambda_{y}\left(\varepsilon^{\prime}\right)$.

ii) If $\alpha_{1} \in \Lambda_{y_{1}}(\varepsilon)$ and $\alpha_{2} \in \Lambda_{y_{2}}(\varepsilon)$, then for all $x \in S_{X_{1}} \times \cdots \times S_{X_{n-1}}$,

$$
\left|\alpha_{1}-\alpha_{2}\right| \leq \frac{2 \varepsilon}{\left|A\left(x, y_{1}\right)\right|}+\frac{2 \varepsilon}{\left|A\left(x, y_{2}\right)\right|}
$$


(where if a denominator is zero, we agree that the fraction equals $\infty$ ).

iii) If $\alpha \in \Lambda_{y}(\varepsilon)$, then $D(\alpha, r) \subset \Lambda_{y}\left(\varepsilon+r\left\|A_{y}\right\|\right)$, where $D(\alpha, r)$ is the closed disc centered at $\alpha$ with radius $r$.

Proof. ii) Suppose $\alpha \in \Lambda_{y}(\varepsilon)$ and $\beta \in \Lambda_{x}(\varepsilon)$. Then

$$
\begin{aligned}
|\alpha-\beta||A(x, y)| & =|\alpha A(x, y)-\beta A(x, y)| \\
& =|\alpha A(x, y)-B(x, y)+B(x, y)-\beta A(x, y)| \\
& \leq|B(x, y)-\alpha A(x, y)|+|B(x, y)-\beta A(x, y)| \\
& \leq\left\|B_{y}-\alpha A_{y}\right\|+\left\|B_{x}-\beta A_{x}\right\| \\
& \leq 2 \varepsilon,
\end{aligned}
$$

so $|\alpha-\beta| \leq \frac{2 \varepsilon}{|A(x, y)|}$. Now let $\alpha_{1} \in \Lambda_{y_{1}}(\varepsilon)$ and $\alpha_{2} \in \Lambda_{y_{2}}(\varepsilon)$, and take $\beta \in \Lambda_{x}(\varepsilon)$, which is non-empty by Lemma 1.2. Therefore,

$$
\left|\alpha_{1}-\alpha_{2}\right| \leq\left|\alpha_{1}-\beta\right|+\left|\beta-\alpha_{2}\right| \leq \frac{2 \varepsilon}{\left|A\left(x, y_{1}\right)\right|}+\frac{2 \varepsilon}{\left|A\left(x, y_{2}\right)\right|} .
$$

iii) Say $\alpha \in \Lambda_{y}(\varepsilon)$ and $|\lambda| \leq r$. We have

$$
\begin{aligned}
\left\|B_{y}-(\alpha+\lambda) A_{y}\right\| & \leq\left\|B_{y}-\alpha A_{y}\right\|+\left\|\lambda A_{y}\right\| \\
& =\left\|B_{y}-\alpha A_{y}\right\|+|\lambda|\left\|A_{y}\right\| \\
& \leq \varepsilon+r\left\|A_{y}\right\|,
\end{aligned}
$$

so $\alpha+\lambda \in \Lambda_{y}\left(\varepsilon+r\left\|A_{y}\right\|\right)$. Thus $D(\alpha, r) \subset \Lambda_{y}\left(\varepsilon+r\left\|A_{y}\right\|\right)$.

The constants $c_{n}$ appearing in the following proof are the constants referred to in the Introduction (see 4, 9 $)$. Also, we use the fact that $k$-linear forms are $k$-homogeneous polynomials. Indeed, any $k$-linear form on $X_{1} \times \cdots \times X_{k}$ is a $k$ homogeneous analytic function on the space $X_{1} \times \cdots \times X_{k}$.

Theorem 1.4. For all $n$, there is a constant $D_{n}$ such that if $A$ and $B$ are $n$-linear forms of norm one, then

$$
Z(A) \subset \varepsilon(B) \Rightarrow\|B-\alpha A\| \leq D_{n} \varepsilon \quad \text { for some }|\alpha|=1 .
$$

Proof. We first observe that by induction on $n$, there exist constants $d_{n}$ such that for such $n$-linear forms

$$
Z(A) \subset \varepsilon(B) \Rightarrow\left\|B-\alpha_{0} A\right\| \leq d_{n} \varepsilon,
$$

for some complex number $\alpha_{0}$. Indeed, the case $d_{1}=1$ follows from the first part of Phelps' Lemma. For $n>1$, suppose the result true for $(n-1)$-linear forms, and proceed as in Lemma 1.2 to obtain that $\Lambda_{y}\left(d_{n-1} \varepsilon\right)$ is non-empty.

Next, take $\delta \in(0,1)$. Choose $y_{\delta} \in S_{X_{n}}$ such that $\left\|A_{y_{\delta}}\right\| \geq 1-\delta$, and take another $y \in S_{X_{n}}$ for which $A_{y} \neq 0$. Since $k$-linear forms are $k$-homogeneous polynomials, there is a constant $c_{n-1}>0$ such that

$$
c_{n-1}\left\|A_{y}\right\|\left\|A_{y_{\delta}}\right\| \leq\left\|A_{y} A_{y_{\delta}}\right\| .
$$

Choose $x \in S_{X_{1}} \times \cdots \times S_{X_{n-1}}$ such that

$$
\left(c_{n-1}-\delta\right)\left\|A_{y}\right\|\left\|A_{y_{\delta}}\right\|<\left|A(x, y) A\left(x, y_{\delta}\right)\right|,
$$


and take $\alpha \in \Lambda_{y}\left(d_{n-1} \varepsilon\right)$ and $\alpha_{\delta} \in \Lambda_{y_{\delta}}\left(d_{n-1} \varepsilon\right)$, which, as we have just seen, are nonempty sets (note that $A(x, y)$ and $A\left(x, y_{\delta}\right)$ are non-zero). Then by ii) of Lemma 1.3 ,

$$
\begin{aligned}
\left|\alpha-\alpha_{\delta}\right| & \leq \frac{2 d_{n-1} \varepsilon}{|A(x, y)|}+\frac{2 d_{n-1} \varepsilon}{\left|A\left(x, y_{\delta}\right)\right|} \\
& =\frac{2 d_{n-1} \varepsilon\left(\left|A\left(x, y_{\delta}\right)\right|+|A(x, y)|\right)}{\left|A(x, y) A\left(x, y_{\delta}\right)\right|} \\
& \leq \frac{4 d_{n-1} \varepsilon}{\left|A(x, y) A\left(x, y_{\delta}\right)\right|} \\
& \leq \frac{4 d_{n-1} \varepsilon}{\left(c_{n-1}-\delta\right)\left\|A_{y}\right\|\left\|A_{y_{\delta}}\right\|}=r .
\end{aligned}
$$

Thus $\alpha_{\delta} \in D(\alpha, r) \subset \Lambda_{y}\left(d_{n-1} \varepsilon+r\left\|A_{y}\right\|\right)=\Lambda_{y}\left(d_{n-1} \varepsilon+\frac{4 d_{n-1} \varepsilon}{\left(c_{n-1}-\delta\right)\left\|A_{y_{\delta}}\right\|}\right)$ by iii) of Lemma 1.3. So

$$
\begin{aligned}
\alpha_{\delta} & \in \Lambda_{y}\left(d_{n-1} \varepsilon+\frac{4 d_{n-1} \varepsilon}{\left(c_{n-1}-\delta\right)(1-\delta)}\right) \\
& =\Lambda_{y}\left(\left[1+\frac{4}{\left(c_{n-1}-\delta\right)(1-\delta)}\right] d_{n-1} \varepsilon\right) .
\end{aligned}
$$

On the other hand, if $y$ is such that $A_{y}=0$,

$$
\mathbb{C}=\Lambda_{y}(\varepsilon) \subset \Lambda_{y}\left(\left[1+\frac{4}{\left(c_{n-1}-\delta\right)(1-\delta)}\right] d_{n-1} \varepsilon\right) .
$$

Thus,

$$
\alpha_{\delta} \in \bigcap_{y \in S_{X_{n}}} \Lambda_{y}\left(\left[1+\frac{4}{\left(c_{n-1}-\delta\right)(1-\delta)}\right] d_{n-1} \varepsilon\right)=K_{\delta} .
$$

These $K_{\delta}$ 's are non-empty compact sets, decreasing as $\delta \rightarrow 0$, so

$$
\bigcap_{\delta>0} K_{\delta} \neq \emptyset
$$

For $\alpha_{0} \in \bigcap_{\delta>0} K_{\delta}$, we have

$$
\begin{aligned}
\left\|B-\alpha_{0} A\right\| & =\sup _{y} \sup _{x}\left|B(x, y)-\alpha_{0} A(x, y)\right| \\
& =\sup _{y}\left\|B_{y}-\alpha_{0} A_{y}\right\| \\
& \leq\left(1+\frac{4}{c_{n-1}}\right) d_{n-1} \varepsilon, \\
& =d_{n} \varepsilon,
\end{aligned}
$$

say. Since $A$ and $B$ have norm one, arguing as in the second part of Phelps' Lemma, with $\alpha=\frac{\alpha_{0}}{\left|\alpha_{0}\right|}$, we get

$$
\|B-\alpha A\| \leq 2 d_{n} \varepsilon, \quad \text { with }|\alpha|=1 .
$$

Thus, letting $D_{n}=2 d_{n}$, the proof is complete.

Note that the constant obtained in the theorem is

$$
D_{n}=2 d_{n}=2\left(1+\frac{4}{c_{1}}\right)\left(1+\frac{4}{c_{2}}\right) \cdots\left(1+\frac{4}{c_{n-1}}\right),
$$


where the $c_{k}$ 's are the constants found in [4. Thus in the complex case

$$
D_{n}=2 \prod_{k=1}^{n-1}\left(1+4^{k+1}\right) \text {, }
$$

while in the real setting,

$$
D_{n}=2 \prod_{k=1}^{n-1}\left(1+2 \cdot 16^{k}\right) \text {. }
$$

\section{REFERENCES}

1. Acosta, M., Aguirre, F., and Payá, R. There is no bilinear Bishop-Phelps Theorem. Israel J. of Math. 93 (1996), 221-228. MR.1380644 (97d:46018)

2. Aron, R., Downey, L., and Maestre, M. Zero sets and linear dependence of multilinear forms. Note di Mat., (1) 25, 2005-2006 (2006), 49-54. MR2220451

3. Bishop, E. and Phelps, R. A proof that every Banach space is subreflexive. Bull. Amer. Math. Soc., 67 (1961), 97-98. MR0123174 (23:A503)

4. Benítez, C., Sarantopoulos, Y., and Tonge, A. Lower bounds for the norms of products of polynomials. Math. Proc. Cambridge Philos. Soc., 124 (1998), 395-408. MR.1636556 (99h:46077)

5. Cardwell, A. A new proof of a Lemma by Phelps, International Journal of Mathematics and Mathematical Sciences, Vol. 2006 (2006).

6. Fabian, M., Habala, P., Hájek, P., Montesinos, V., Pelant, P., and Zizler, V. Functional Analysis and Infinite-Dimensional Geometry. CMS Books in Mathematics. Springer-Verlag, 2001. MR 1831176 (2002f:46001)

7. Fabian, M., Montesinos, V., and Zizler, V. A characterization of subspaces of weakly compactly generated Banach spaces. J. London Math. Soc. (2) 69 (2004), 457-464. MR2040615 (2004k:46026)

8. Phelps, R. A representation theorem for bounded convex sets. Proc. Amer. Math. Soc., 11 (1960), 976-983. MR 0123172 (23:A501)

9. Ryan, R. and Turett, B. Geometry of spaces of polynomials. Jour. of Math. Analysis and Appl., 221 (1998), 698-711. MR.1621703 (99g:46015)

Department of Mathematical Sciences, Kent State University, Kent, Ohio 44242

E-mail address: aron@math.kent.edu

Mathematics Department, Millersville University, P.O. Box 1002, Millersville, Pennsylvania 17551-0302

E-mail address: Antonia.Cardwell@millersville.edu

Departamento de Análisis Matemático, Universidad de Valencia, 46100 Burjassot, VAlencia, Spain

E-mail address: domingo.garcia@uv.es

Depto. de Matemática, Universidad Torcuato Di Tella, Miñones 2159/77 (C1428ATG), Buenos Aires, Argentina

E-mail address: nacho@utdt.edu 